\title{
Erratum to: The application of Lie point symmetries to the resolution of certain problems in financial mathematics with a terminal condition
}

\author{
J. G. O’Hara · C. Sophocleous · P. G. L. Leach
}

Received: 10 December 2014 / Accepted: 10 December 2014 / Published online: 9 January 2015

(C) Springer Science+Business Media Dordrecht 2015

\begin{abstract}
In an analysis of the Lie point symmetries of the equation which allows for the inclusion of transaction costs into the Black-Scholes model (J Eng Math 82:67-75, 2013) one of the symmetries was omitted. We rectify that failing and demonstrate that the correctness of the subsequent analysis is not compromised.
\end{abstract}

Keywords Evolution partial differential equations · Financial mathematics $\cdot$ Lie symmetries

Mathematics Subject Classification $35 \mathrm{~K} 05 \cdot 60 \mathrm{H} 05 \cdot 91 \mathrm{G} 80$

Erratum to: J Eng Math (2013) 82:67-75

DOI 10.1007/s10665-012-9595-4

The governing evolution partial differential equation for the Black-Scholes model when transaction costs are included is [1] (their Eq. 3.1)

$0=u_{t}+\frac{1}{2} \tilde{\sigma}^{2} x^{2} u_{x x}+b \sigma^{2} x^{3} u_{x x}^{2}+r\left(x u_{x}-u\right)$

subject to the terminal condition

$u(T, x)=f(x)$,

where $f(x)$ is initially unspecified and is to be determined from the analysis.

The online version of the original article can be found under doi:10.1007/s10665-012-9595-4.

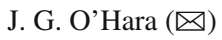

CCFEA, University of Essex, Wivenhoe Park CO4 3SQ, England

e-mail: johara@essex.ac.uk

C. Sophocleous · P. G. L. Leach

Department of Mathematics and Statistics, University of Cyprus, Nicosia, Cyprus

e-mail: christod@ucy.ac.cy

P. G. L. Leach

e-mail: leachp@ukzn.ac.za; leach@ucy.ac.cy

P. G. L. Leach

School of Mathematical Sciences, University of KwaZulu-Natal, Durban, Republic of South Africa 
We analyse (1) for the possession of Lie point symmetries. We find that in general there are five Lie point symmetries, namely

$\Gamma_{1}=\partial_{t}, \quad \Gamma_{2}=x \partial_{u}, \quad \Gamma_{3}=e^{r t} \partial_{u}, \quad \Gamma_{4}=x \partial_{x}+u \partial_{u}$,

$\Gamma_{5}=t \partial_{t}+r t x \partial_{x}+\left\{\frac{\tilde{\sigma}^{2} x}{8 b \sigma^{2}}\left(2+2 r t+\tilde{\sigma}^{2} t-2 \log x\right)+(r t-1) u\right\} \partial_{u}$.

To solve the boundary-value problem we apply the general symmetry, $\Gamma=\sum_{i=1}^{5} \alpha_{i} \Gamma_{i}$, where the $\alpha_{i}, i=1, \ldots, 5$, are as yet arbitrary constants, to the terminal conditions (2). We obtain the two relations

$\alpha_{1}+\alpha_{5} T=0$

and

$\alpha_{2} x+\alpha_{3} e^{r T}+\alpha_{5} \frac{\tilde{\sigma}^{2} x}{8 b \sigma^{2}}\left(2+2 r T+\tilde{\sigma}^{2} T-2 \log x\right)+\left(\alpha_{4}+\alpha_{5}(r T-1)\right) f=\left(\alpha_{4}+\alpha_{5} r T\right) x f^{\prime}$.

Note that we have eliminated $\alpha_{1}$ in favour of $-\alpha_{5} T$. The second relation may be considered as a first-order equation for $f(x)$. If $\alpha_{5} \neq 0$, its solution is, obtained using Mathematica,

$$
\begin{aligned}
f(x)= & -\frac{\alpha_{3} \mathrm{e}^{r T}}{\alpha_{4}+\alpha_{5}(-1+r T)}+\frac{\left(8 \alpha_{2} b \sigma^{2}+\tilde{\sigma}^{2}\left(2 \alpha_{4}+\alpha_{5}\left(2+4 r T+\tilde{\sigma}^{2} T\right)\right)\right) x}{8 \alpha_{5} b \sigma^{2}} \\
& +c_{1} x^{1-\alpha_{5} /\left(\alpha_{4}+\alpha_{5} r T\right)}-\frac{\tilde{\sigma}^{2} x \log x}{4 b \sigma^{2}},
\end{aligned}
$$

in which a constant term,

$$
\left(\alpha_{4}+\alpha_{5} r T\right)^{1-\alpha_{5} /\left(\alpha_{4}+\alpha_{5} r T\right)},
$$

has been incorporated into the constant of integration to give $c_{1}$, and, if $\alpha_{5}=0$, we have

$f(x)=c_{1} x-\frac{\alpha_{3}}{\alpha_{4}} e^{r T}+\frac{\alpha_{2}}{\alpha_{4}} x \log x$

where $c_{1}$ is the constant of integration. The form of $f(x)$ in (5) provides the solution (3.8) in [1], while the form in (4) will be examined elsewhere.

Acknowledgments We thank Professors Sergii Kovalenko and Olena Vaneeva of the Institute of Mathematics in National Academy of Sciences of Ukraine for drawing to our attention the missing symmetry, $\Gamma_{5}$.

\section{Reference}

1. O'Hara JG, Sophocleous C, Leach PGL (2013) The application of Lie point symmetries to the resolution of certain problems in financial mathematics with a terminal condition. J. Eng. Math. 82:67-75. doi:10.10007/s10665-012-9595-4 